\title{
The Softurator: When, Where and How We do It?
}

\author{
${ }^{1}$ Pratik Dipak Shah, ${ }^{2}$ Srijon Mukherji
}

\begin{abstract}
Factors that affect management of surgical defects are surgical site, amount of remaining support tissue, presence of teeth and defect characteristics. When these factors are not favorable, successful prosthetic management of surgical defect becomes more challenging. Simultaneously, prosthesis should be comfortable to supporting and surrounding tissues as well as surgical defect. In the presented case, we have used soft polyvinyl siloxane material for fabrication of temporary surgical obturator to cover the surgical defect at the junction of hard and soft palates. Soft, flexible material is used for better adaptation to the changing morphology of surgical defect and also improves functions like speaking and swallowing.
\end{abstract}

Keywords: Flexible, Polyvinyl siloxane, Surgical obturator.

How to cite this article: Shah PD, Mukherji S. The Softurator: When, Where and How We do It? Int J Head Neck Surg 2015; 6(2):93-95.

\section{Source of support: Nil}

Conflict of interest: None

\section{INTRODUCTION}

Surgical resection is an established and common method for treatment of maxillofacial tumors. Such intervening surgery in this region may involve the destruction of oral, antral or nasal mucosa. At the end, surgical defect may affect speech, swallowing and mastication. After surgical resection, bigger job is left to rehabilitate the patient as close as possible so that a person enjoys in good health. There are two broad categories available for the rehabilitation of the surgical defect: prosthetic obturators and flap reconstruction. Obturators have an advantage of shortening operating room time and eliminating flap donor side morbidity. ${ }^{1}$ The primary role of a temporary surgical obturator is to aid in healing during recovery period and shorten long-term convalescence and rehabilitation. It supports soft tissues, thus, minimizing scar contracture, protects the wound from trauma and contamination with food debris. ${ }^{2}$ As with surgical obturator, patients are often able to tolerate a liquid diet from the first postoperative

\footnotetext{
${ }^{1}$ Surgical Fellow, ${ }^{2}$ Director and Chief Consultant

1,2Department of Oral and Maxillofacial Surgery, Calcutta Institute of Maxillofacial Surgery and Research, Kolkata, West Bengal, India

Corresponding Author: Pratik Dipak Shah, Surgical Fellow Department of Oral and Maxillofacial Surgery, Calcutta Institute of Maxillofacial Surgery and Research, Kolkata, West Bengal, India Phone: 03324412366, e-mail: pratikdshah.2711@gmail.com
}

day. ${ }^{1}$ In this case report, we describe the use of polyvinyl siloxane material in the fabrication of temporary surgical obturator to rehabilitate surgical defect following tumor resection at the junction of hard and soft palates which passively adapts to changing palatal and velopharyngeal morphology during function.

\section{CASE REPORT}

A 56 years old male patient reported to Calcutta Institute of Maxillofacial Surgery and Research Centre with the chief complain of nodular ulcerative lesion over the palate since one and half month. On examination, solitary lesion was found which was round in shape measuring $1 \times 1 \mathrm{~cm}$ in diameter. Lesion was located at the junction of hard and soft palates and it was freely movable (Fig. 1). There was no evidence of local, regional or distant lymphadenopathy or evidence of primary tumor elsewhere. Lesion was firm and nontender in nature. The lesion was smaller in size and we suspected it as a benign mass due to above-mentioned reasons. Therefore, excisional biopsy with closed margins ( $5 \mathrm{~mm}$ ) under local anesthesia was performed. Preoperatively, we made impression of maxillary arch with irreversible hydrocolid material (alginate) and transferred it to cast. We performed a mock surgery over the cast and fabricated a temporary surgical obturator using polyvinyl siloxane material which was heated and vacu pressed onto patient's cast. We resected the lesion keeping $5 \mathrm{~mm}$ of margins all around it. The resultant defect involved only the palatal mucosa (Figs 2 and 3). Immediately postoperatively, zinc oxide eugenol

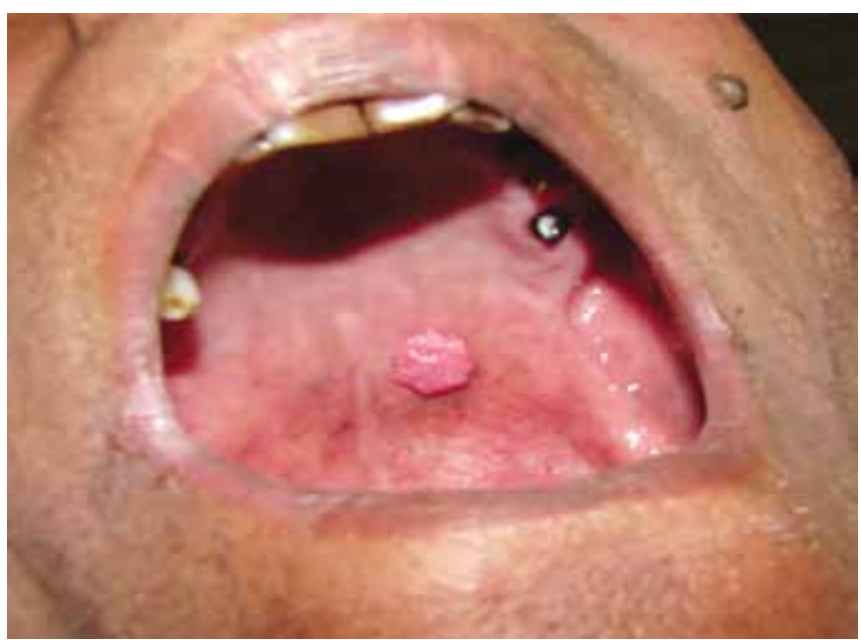

Fig. 1: Ulcerative nodular lesion at the junction of hard and soft palates 
(ZOE) paste which acts as soothing agent was applied to surgical defect and over which temporary surgical obturator was inserted and secured to maxillary arch with Vicryl 3-0 suture (Fig. 4). Patient was followed up at regular interval (Fig. 5). We advised patient to wear prosthesis for a period of 6 weeks till secondary healing is completed. ${ }^{3}$ Histopathological examination revealed a lesion as verrucous hyperplasia. Functional assessment of soft palate was carried out at 3rd and 6th weeks postoperatively. Patient was asked to produce velar sounds which include English ' $G$ ' and ' $K$ '. While producing velar sound body of the tongue meets soft palate. Patient was able to pronounce velar sounds clearly without any discomfort. Swallowing pattern was assessed by seeing patient's reaction to food, observing oral movements in chewing, coughing, struggle behaviors, duration of meal and total intake, coordination of breathing and swallowing. All these parametres were found to be satisfactory.

\section{DISCUSSION}

The main problem of rigid palatal prosthesis is lack of synchronization with the soft palate extension with the

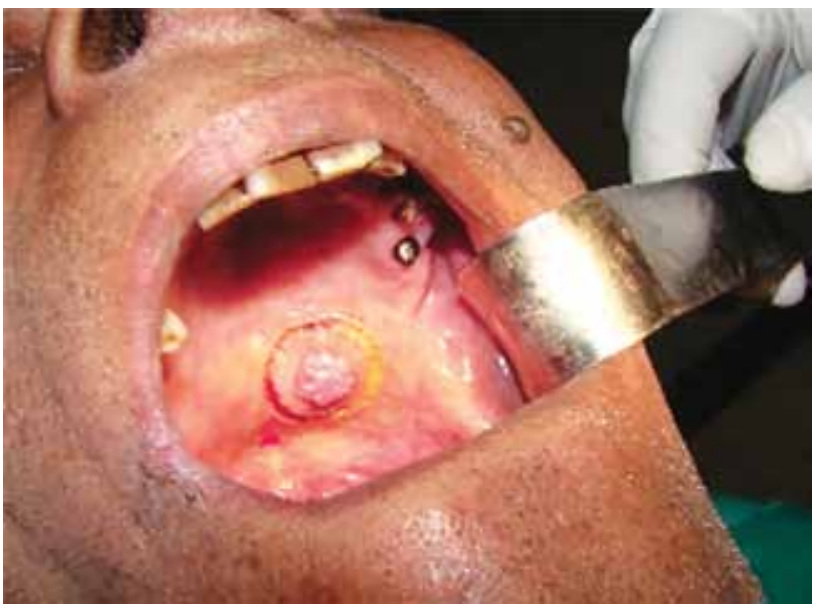

Fig. 2: Keeping $5 \mathrm{~mm}$ of margin all around the lesion during excision (close margin)

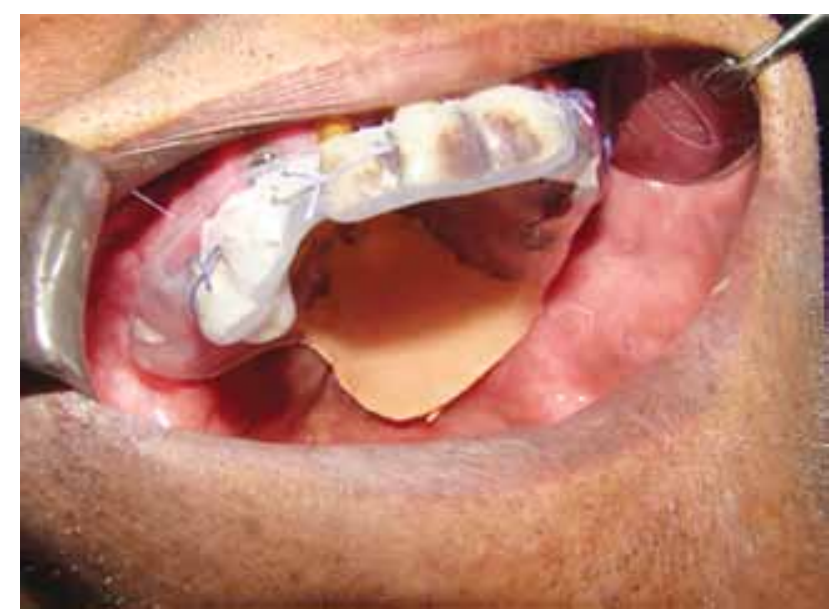

Fig. 4: Soft obturator (Softurator) inserted over the defect along with zinc oxide eugenol paste muscles neighbouring the defect during swallowing. Rigid conventional palatal prosthesis can not adapt to the changing morphology of the surgical defect. Both the tolerance of the patient and ideal palatal seal are jeopardized. ${ }^{3}$ The soft polyvinyl siloxane material is chosen for its flexibility so it becomes atraumatic to the musculature of the soft palate while swallowing or speaking. That is why we have given the term 'Softurator' (the soft obturator). The disadvantage of this prosthesis was lack of retention and stability; so, in the present case, we had to secure the prosthesis with remaining teeth and alveolar mucosa using vicryl 3.0 suture. Advantage of softurator with $\mathrm{ZOE}$ paste over leaving a defect raw is, $\mathrm{ZOE}$ acts as a soothing agent during healing process. Though palatal prosthesis did not allow inspection of postoperative raw wound, it covered the surgical site and prevented the wound getting traumatized during chewing and swallowing which leads to uneventful healing during postoperative period. Though surgical defect could have also been well reconstructed using palatal rotationl flap based on greater palatine vessel of opposite side; however, it would have required additional locoregional surgical

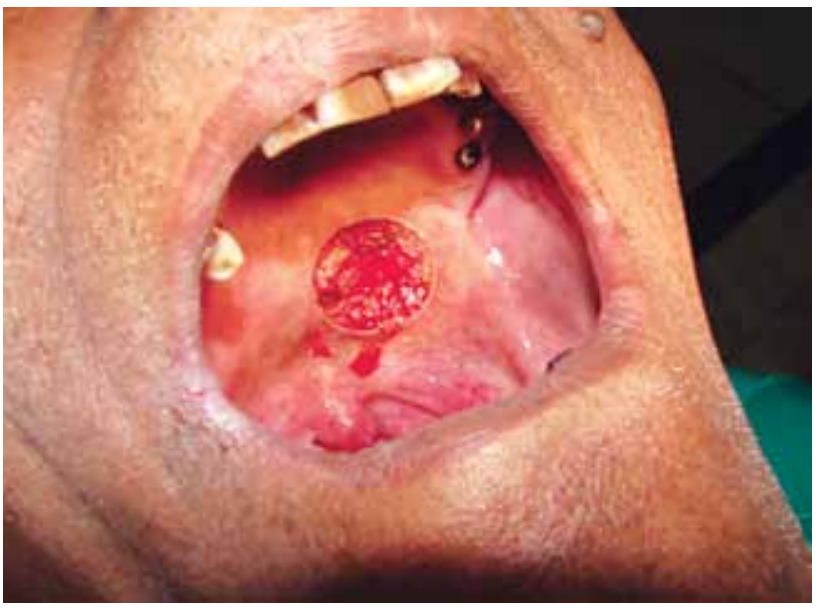

Fig. 3: Surgical defect following wide local excision

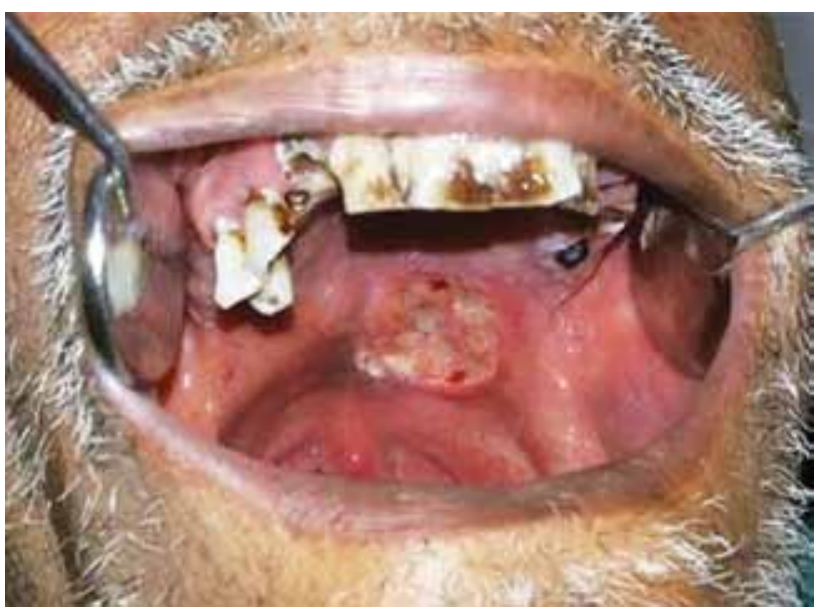

Fig. 5: Healing in progress (1 week postoperatively) 
site and second stage surgical intervention. Considering the additional surgical site morbidity, cost factor and based on patient's choice, soft obturator was taken into consideration to cover the surgical defect.

\section{CONCLUSION}

Maxillofacial defects are highly individual and require the clinician to call upon all his knowledge and experience to fabricate a functional prosthesis. Lot of functional problems may arise if appropriate prosthetic material or proper design is not taken into consideration while fabricating prosthesis. Patient's esthetic and functional requirements must be taken into consideration for good quality of life. The restorative clinician has to be imaginative and innovative and adhere to principles of rehabilitation while designing prosthesis.

\section{ACKNOWLEDGMENT}

We wish to acknowledge Mr Mangal for his valuable technical help in fabricating the prosthesis.

\section{REFERENCES}

1. Ducic Y. An effective, inexpensive, temporary surgical obturator following maxillectomy 2001 Feb;111(2):356-358.

2. Tannamala PK, Pulagam M, Pottem SR, Karnam S. Flexible resins in the rehabilitation of maxillectomy patient. Ind J Dent Res 2012 Jan-Feb;23(1):97-100.

3. A Siddaraju, et al. Basal cell adenoma of palate-report of a rare lesion. AOSR 2013;3(1):51-55. 